ticularly obnoxious to rheumatic affections of joints, though not of the acute form. Pleurodynia and rheumatic cough frequently occur in all ages of childhood. On the contrary, I find they are not disposed to endocardial rheumatism; at least, according to what $I$ have seen, it can be but of rare occurrence in the first years of life. This fact, and the analagous one, concerning the acute general articular form, may perhaps concert in some way to advance the pathological knowledge of the rheumatic dyscrasy. The disposition once acquired may last for years and years. In some children, however, it ceases entirely as they grow older and stronger towards puberty. In some others, of course, there is no trace of it.

As the cause, everybody recognises a sudden change from warm to cold, particularly when the skin is perspiring and damp, cold more than dry. The sudden occurrence of east winds is also very productive of rheumatism According to this property some countries represent a more fertile soil for this malady. In this respect there may be fe $x$ countries worse than Hungary and Austria. England, particularly the eastern parts, enjoys also the discredit of being much troubled with rheumatism.

But, it cannot be denied, the mentioned changes of temperature are, as well, productive of catarrhal and parenchymatous inflammations. There must, therefore, be another condition or circumstance besides, special in prodncing rheumatism instead of pneumonia or catarrh. The electric influence, I believe, is too little known and valued in this respect. I remember many children who, without being exposed to cold, before stormy weather, or some other sudden change of the weather, became affected with rheumatism; and some even when confined to bed, and guarded carefully against " taking cold."

As a prophylactic measure, nothing is more proper than to accustom children, judiciously and by degrees, to the vicissitudes of temperature; performing cold sponging of their whole body, strengthening the more obnoxious kind of skin by lotions of a mixture of brandy and water. The custom to dress children lightly, with bare neck, short stockings, and trousers, as a general measure, is also very suitable, in constitutions which allow it.

Amongst complications, like as in adults, the rheumatic affections frequently combines with the bilious, the catarrhal, and others; the liability of children to rheumatism during the last stage of, or convalescence from, measles, is, however, very striking. But rheumatism combines also occasionally with other eruptive fevers.

The combination of intermittent fever with rheumatism is, I find, interesting. I have had to attend, at Pesth, many children at once affected with both diseases. The latter was only rheumatalgia, more commonly costal pleurodynia, or rheumatic cough, always remittent or even intermitttent, and increasing along with the paroxysm. I do not remember one single case in which pleurodynia, or articular rheumatism, when complicating ague, would have passed into inflammation, swelling, or exudation; nor do I know a single case of genuine inflammation complicating ague. Now let me add to this that I did not see phlegmonous or parenchymatous inflammations, $f$. e., pneumonia, suppurative pleurisy, acute meningitis with plastic or purulent products, in connection with an unequivocallyestablished rheumatic fever, not even with the acutest articular form, which we generally look at as inflammatory, and in which, no doubt, certain degrees and kinds of inflammatory action take place. These are, I believe, facts of some pathological interest. The remissions of the rheumatic fever come sometimes near the intermissions of ague. It stands between the inflammatory and ague.

The most frequent combination of local rheumatism is that with inflammation; thence a modification of the inflammatory process and its products arises, which is not sufficiently studied yet. I have seen only one or two cases of acute arthritis in the knee pass into suppuration; and even in these cases I could not state with confidence that it was originally rheumatism.

An intimate amalgamation of the rheumatic inflammation with the scrofulous dyscrasy, is a common and occasionally sad occurrence in childhood.

Those that have penetrated a little deeper into the difficulties of children's practice, and those of you who intend to do so, will perhaps excuse the minuteness and length with which $I$ have dwelt on the subjects of this lecture. There are some obvious things which it is still suitable to call to mind in various practical connections. I will next speak of the treatment.

\section{ON THE TREATMENT OF CHOLERA} BY CALOMEL AND COLOCYNTH.

\section{Bi JOIIN JONES, M.R.C.S., ENG., Derby.}

WhATEver may be the nature of the poison which produces this terrible pestilence, or by whatever laws it traverses various regions, countries, and districts, and propagates its pestiferous influence, its primary action is, doubtless, on the nervous and vascular systems; but whether these are simultaneously affected, or in succession, is uncertain. Nervous depression, speedily followed by vascular congestions, particularly of the large veins of the abdominal viscera, seems to constitute the first stage. From the peculiar nature of the choleraic poison, of which nothing certain is known, the important functions of absorption and secretion become suspended, and in some organs altogether stopped. The mucous membrane of the intestinal tube throughout seems to be deprived of its usual secreting and absorbing functions, the mouth and fauces are no longer supplied with their natural moisture, and unquenchable thirst is the consequence. The mucous secretion of the stomach, and of the large and small intestines, is suspended, and seems superseded by a frightful exudation or effusion of the serum of the blood, constituting the copious watery evacuations, both by romiting and purging, which for the most part are so characteristic of the 
disease; mixed with these is, probably, the chyle, । which the lacteals, deprived of their natural function, are unable to absorb, giving the evacuations the peculiar and characteristic appearance of rice-water. The liver ceases to secrete bile; for although in post-mortem examinations bile has been found in the gall-bladder, none flows into the duodenum. The kidneys secrete no urine, none being found in the bladder, which is usually contracted and empty.

The blood, deprived of its serum, has generally been found to be "black, or dark-coloured, not unlike tar in its consistence, thick, ropy, and semi-coagulated." This state of the blood, together with congestion of the lungs, preventing the usual oxygenation of the blood, sufficiently accounts for the blue colour of the skin, which in some fatal cases becomes nearly black. Arising from defective oxygenation of the blood, and impeded secreting functions, occurs also the reduced temperature, which is so remarkable as to cause the surface of the body to feel as cold as marble, and even the tongue and breath to feel cold.

The symptoms indicating amendment arise from the restoration of the absorbing and secreting functions of the abdominal viscera. Thus the appearance of bile and foculency in the alvine dejections are the harbingers of safety. Simultaneously with this gratifying occurrence, the symptoms of collapse disappear; the characteristic, watery, and light-coloured evacuations become gradually less liquid, frequent, and abundant; cramps subside; thirst is no longer urgent; the pulse rises; the temperature of the skin is restored, accompanied with a warm perspiration; the renal secretions are resumed, and for the first time from the commencement of the attack, micturition occurs.

The same causes which predispose to the procuction and spread of typhus fever, are also powerful in the production of cholera, when that peculiar and mysterious agency on which it more immediately depends, exists in the atmosphere. These are :-

1. Constitutional peculiarity, which renders some persons naturally more susceptible of the disease than others.

2. Great bodily fatigue, or mental depression.

3. Defective nutrition, arising either from want of nourishment, or from impaired digestion, by which due assimilation of the blood is prevented.

4. A vitiated state of the air, produced by exhalations from putrid animal and vegetable matters, abundantly generated by local nuisances, such as open cess-vaults, pigsties, slaughter-houses, \&c. ; badly ventilated houses, such as are generally occupied by the poor in back streets, courts, and alleys, and are often found built back to back, as if on purpose to prevent free circulation of the air; filthy, crowded, and badly-ventilated apartments, in which the inhabitants are constantly breathing the noxious air arising from their own lungs in respiration, rendered still more noxious by want of personal cleanliness.

In proportion as individuals have been exposed to the above predisposing causes of the disease during the prevalence of epidemic cholera, will be their liability to become affected.

In the autumn of 1849 the inhabitants of Derby were thrown intogreat consternation by a visitation of cholera. In anticipation of its approach, every preparation was made to check its progress, and restrain its ravages, On its arrival the town was divided into districts, and a resident medical practitioner was appointed by the Board of Guardians to undertake the medical treatment of all cases which might occur amongst the poor in his district, and that there might not be unnecessary delay in the treatment, brandy, or whatever else might be required, was obtained by a written order from the medical attendant, addressed to any neighbouring shop, at the expense of the Guardians. This laudable plan was continued for about three weeks, but in consequence of the expense being thought too great, it was discontinued. In the district attended by my partner and myself, including several back streets and courts thickly inhabited by the poor, besides cases of dysentery, which was at the same time prevalent, thirty-two cholera cases were treated, and cured, who were affected with rice-water evacuations, together with cramps and symptoms of rapid exhaustion, some of whom were in a state of advanced collapse, with pulse hardly perceptible, surface cold as a corpse, breath and tongue cold, features shrunk, and marked blueness of the skin. The disease in nearly all the cases treated was preceded by neglected diarrhœa, which, after continuing for a few days, suddenly assumed the peculiar character of cholera.

Treatment.-The leading indication in the treatment of this fearful malady, is to restrain the escape of serum from the blood, on which its peculiar and fatal malignity depends. If this important indication can be fulfilled, speedy amendment is the consequence, if not, the progress of the disease becomes fearfully rapid, and certainly fatal, a few hours only deciding the momentous question whether it will terminate in death or recovery.

As diarrhœe usually precedes an attack of cholera, it becomes a matter of the utmost importance to distinguish between this state and actual cholera, in which it may or may not terminate; more particularly as the remedies which are found most appropriate in the one, are diametrically opposed to those which are found most efficient in the other. Chalk mixture, astringents, and opiates, which are so necessary and beneficial in diarrhoea, having no other effect than hastening the fatal catastrophe in cholera. Amongst the multitudinous remedies which have been recommended in the treatment of this fatal disease since its appearance in this country, those which are more or less purgative have been found most beneficial. Amongst these calomel has always been considered pre-eminent. This invaluable remedy in conjunction with the compound extract of colocynth I have found invariably successful, if administered sufficiently early, and before the occurrence of fatal collapse. The direct effect of this combination is to prevent the further escape of serum from the blood, by restoring the functions of the mucous 
membrane of the alimentary canal, and in consequence, the functions of the abdominal viscera generally. Pereira, in his "Elements of Materia Medica," says :- "Colocynth, taken in small or moderate doses, acts as a very safe and useful purgative. Its operation is not limited to the acceleration of the vermicular movements, but is extended to the secreting and exhaling vessels of the alimentary canal, whose functions it promotes. Moreover, it stimulates the other abdominal organs, and after the absorption of its bitter acrid principle, it not unfrequently proves diuretic." "These remarks apply to the compound extract, the only preparation of colocynth of which I have personal experience. It would appear partly from observation in the human subject, and also from the experiments of Orfila on dogs, that colocynth is one of those purgatives which exert a specific stimulant influence over the large intestines."

My mode of treatment was as follows:-The abdomen and upper and lower extremities being first well rubbed with turpentine liniment, the patient was immediately wrapped in flannel steeped and wrung out with hot water, over which, and covering the whole body as if swaddled, was applied a warm dry blanket. This fomentation, \&cc., being repeated every two hours, two of the following pills were directed to be taken immediately, and one repeated every hour till the motions became feculent and more natural.-R. Chlorid. Hydrarg., gr. viij.; Extr. Colocynth. Comp., gr. xij; Ol. Carui, gtt. iij. M. Ft. Pil., viij. If the pills were rejected they were immediately repeated and occasionally accompanied with brandy or hot brandy and water.

Under this treatment a manifest amelioration of all the symptoms speedily occurred; the vomiting became less urgent; the alvine dejections instead of being more frequent as might, a priori, be expected as the effect of the purgative pills, becomes invariably less frequent, less copious, of greater consistency, and in a few hours feculent and mixed with bile. When these gratifying changes in the evacuations occurred, speedy and uninterrupted recovery usually followed.

The suddenness of the recovery of patients from being almost apparently in articulo mortis, was very remarkable, and in one instance was considered by the friends of the individual, who were "Mormonites or Latter-day Saints," an actual miracle, which they published in one of their tracts as a notable instance of the result of their prayers and laying on of hands! In some cases, however, after the cholera symptoms had subsided, slight fever occurred, which continued only for a few days.

It is a fact worthy of remark, that out of the thirtytwo cases of cholera attended, eleven occurred on one side of Agard Street, besides nine cases of dysentery. This is a short street behind which a filthy brook runs, part of which is dammed up for the supply of mills and is generally stagnant; the remainder is very shallow and contains all kinds of noxious refuse. On the other ide of the street, into part of which two large silks factories open, and the remainder is open to gardens, not a single case was reported.

When cholera first made its appearance in this country, Derby was not exempt from its visitation, but compared with other towns its ravages were not great. More than half the cases reported, however, proved fatal. After the epidemic ceased solitary instances occasionally occurred, having all the peculiar characters of the Asiatic disease, which were equally rapid in their progress and fatal in their termination unless arrested by timely and appropriate treatment. Since its last visitation to Derby, in 1849, when its ravages were much greater than on the former occasion, solitary cases have also occurred, having so much the character of Asiatic cholera, that if the disease had at the same time appeared as an epidemic, they would without hesitation have been reported as such. I select the following out of eight cases which have been successfully treated by myself and partner, one of which occurred in October, 1850 , a year after the epidemic had ceased, the other in August last.

Case 1.-Ann Jackson, aged 50, after having been affected with diarrhœa for three weeks, was suddenly seized on Monday morning, October 22, 1850, with vomiting, almost incessant purging, and cramps of the arms and legs. Motions passed involuntarily, thin, watery, and light coloured, resembling rice-water. Saw her at nine o'clock A.M., surrounded hy her family, who were in deep distress on account of what they considered her hopeless condition, lying on her back, mouth half open; tongue furred and feeling cold to the touch; eyes sunk in their sockets; voice scarcely audible; nearly pulseless; skin of a dark colour and cold as a corpse. Although apparently in a complete state of collapse, she was perfectly sensible. The abdomen, arms and legs, were directed immediately to be rubbed with turpentine liniment and covered with flannels wrung out with hot water, and then to be wrapped or swaddled in a dry blanket. To be repeated every two hours.-R. Chlor. Hydrarg., gr. viij.; Extr. Colocynth. Comp., gr. xiij; Sap. Ven., gr., v.; Ol. Carui, gtt. iij. M. Ft. Pil. viiij. Two to be taken immediately, and one every hour till the motion becomes natural. Also to take every half hour brandy in sago.

5, P.M.-Is much warmer; has had no return of vomiting or purging, and cramps but very slightly.Pergat.

Wednesday, 10 A.M.-Has taken the pills regularly, except when asleep; slept for several hours during the night; has had but one motion, not so thir or copious as they have been, but still white.-Pergat.

Thursday.-In much the same state as yesterday; has had a tolerable night; one or two motions (white); reaction fully developed; tongue dry, and much furred.-R. Liq. Ammon. Aciet., oz. ij. ; Sp. Eth. Sulph. Comp., dr. iij.; Mist. Camph., oz. vss. M. Cap. oz. j., quartis horis. Pills to be repeated every two hours.

Friday.-Has had a good night, and this morning has passed several dark-coloured motions, and micturated for the first time since Sunday last.

Saturday.-Is much better in all respects; motions have become of a natural colour and consistency.-To take the pills once or twice a-day. 
Monday.-Continues convalescent, and feels little more than general feebleness, from which she soon recovered.

Case 2.-Mr. Harrey, clerk of St. Werburgh's Church, aged 54, states that on rising in the morning of Monday, the 29th of August, he was affected with diarrhœa, accompanied with general lassitude, slight pain of the bowels, and faintness; motions very frequent, liquid, and copious. Had taken on the preceding night eel pie for supper, of which he says he eat very sparingly. The whole of Monday diarrhœa continued, with little abatement, for which he repeatedly took brandy. Tuesday morning he had a rhubarb draught, and occasionally took brandy ; felt better, and walked about the town transacting business; in the afternoon motions much more frequent, copious, and liquid, with increased faintness. He continued occasionally to take brandy. About 11 o'clock, P.M., on getting out of bed, was seized with cramps of the arms and legs, fell down, and was unable to rise ; whilst on the floor had a copious and involuntary motion, passed with great force, and he became extremely faint. On being lifted into bed I was immediately sent for. I found him greatly exhausted, affected with cramps of the upper and lower extremities, extending to the fingers and toes, and across the chest, excited and aggravated by the slightest movement. He had just vomited nearly half-a-potful of dark-coloured watery fluid; pulse full and slow; skin moderately warm, but of a darker colour than usual; eyes surrounded with a dark-coloured areola, and sunk in their sockets.

His feet were directed to be immediately placed in hot water and mustard; the abdomen and extremities to be rubbed with turpentine liniment, and afterwards a flannel, wrung out with hot water, to be applied; he was then wrapped round, as if swaddled, in a warm dry blanket. To be repeated every two hours. Two of the following pills were immediately given him, and one directed to be repeated every hour till natural motions were produced.-R. Chlor. Hydrarg., gr. viij.; Extr. Colocynth Comp., gr. xij.; Camph., gr. viij.; Ol. Carui., gr.oij. M. Fiat Pil. viij.

I remained with him till about five o'clock, and left him in an improved state, although during my stay be had frequently romited copiously thin, clear, and liquid matters, and passed one or two motions, also copious, resembling milk-and-water, or rather the characteristic rice-water evacuations. Thirst was incessant and unquenchable. Cramps continued, but not so violent. About an hour after having left him, I was sent for in great haste, as the cramps had returned so severely that he thought he could not live; I found him, however, much better, although the cramps had been very severe, in consequence of getting out of bed. $\mathrm{He}$ was in a profuse perspiration; the change in the motions was more marked, less liquid, and not quite so white, although still abundant; cramp less serere; vomiting still copious, but not so frequent. The pills to be continued without the camphor, after which the vomiting ceased. In the course of the day (Wednesday) the motions became more feculent, and slightly mixed with bile, accompanied with corresponding amendment.

Thursday.-Had a good night; motions much more natural, and for the first time since Tuesday has passed urine. The pills were omitted, and the following mixture substituted.-R. Pulv. Chlor., scr. ij.; Mag. Carb., dr. ss.; Mucil. Gum. Acaciæ, oz. ss.; Sp. Ammon. Arom., Tinct. Hyoscy. utrq., dr. ij. ; Aquæe Menth., oz. v. M. Cap. oz. j., bis terve die.

From this time convalescence became rapid, and on Saturday he left his room complaining of little more than weakness.

Total amount of calomel taken sixteen grains, and of compound extract of colocynth thirty-two grains.

October 9, 1852.

\section{EXTRANEOUS SUBSTANCES IN THE EYE.}

\section{By AUGUSTIN PRICHARD, Esq., Bristol.}

Bead before the Bath and Bristol Branch of the As8ociation, October 7, 1852.

THE detection and treatment of the symptoms produced by foreign bodies in the eye, are matters of consideable surgical interest, dependent upon the value of the organs implicated, the destructive results of inefficient surgical aid, and the thoroughly satisfactory cures obtained by the most simple measures. Besides this, the subject is a surgical curiosity. Having constantly under my care a number of these cases, I have for ten years past always examined with a microscope the minute bodies which $I$ have removed from the surface of the eye, and as might perhaps be supposed, have met with a considerable variety.

It will perhaps make my paper more worthy of a scientific body like the present, if I first give some account of the numbers of accidents to the eyes, in proportion to the whole number admitted to an ophthalmic charity, of the per centage of accidents where a foreign substance still remains in the eye at the period of admission, of the numbers of the different kinds of substances found, and the occupation of the applicants. I will afterwards show to any who feel disposed to examine them, some of the objects I have prepared.

My observations are collected at the Bristol Dispensary for the cure of complaints in the eyo, founded by $\mathrm{Mr}$. Estlin in 1812; and they go back as far as the year 1834, from which time the various injuries have been more distinctly arranged under their separate heads than they were before. This comprises a period of eighteen years, during the first part of which I attended the Eye Dispensary as pupil, and for the last ten years as surgeon.

Thirty-seven thousand one hundred and ninety-nine patients have been admitted during this time, that is about 2100 in every year, upon an arerage. 2856 of these cases were injuries to the eye, being between seven and eight in erery hundred. 